Georgian Mathematical Journal

Volume 12 (2005), Number 4, 763-768

\title{
PREIMAGES OF RESIDUAL SETS OF CONTINUOUS FUNCTIONS UNDER OPERATION OF SUPERPOSITION
}

\author{
ARTUR WACHOWICZ
}

\begin{abstract}
Let $C=C[0,1]$ denote the Banach space of continuous real functions on $[0,1]$ with the sup norm and let $C^{*}$ denote the topological subspace of $C$ consisting of functions with values in $[0,1]$. We investigate the preimages of residual sets in $C$ under the operation of superposition $\Phi: C \times C^{*} \rightarrow C, \Phi(f, g)=f \circ g$. Their behaviour can be different. We show examples when the preimages of residual sets are nonresidual of second category, or even nowhere dense, and examples when the preimages of nontrivial residual sets are residual.
\end{abstract}

2000 Mathematics Subject Classification: 26A15, 54E52.

Key words and phrases: Baire category, residual set, space of continuous functions, superposition.

In what follows $C$ will denote the Banach space of continuous real functions on $[0,1]$ with the sup norm, and $C^{*}$ will denote the topological subspace of $C$ consisting of functions with values in $[0,1]$. Observe that $C^{*}$ is a closed subset of $C$, therefore is also a complete space. Let $B(f, \varepsilon)$ and $B_{*}(g, \sigma)$ denote open balls in $C$ and in $C^{*}$, respectively. In [6] (see also [1]) we have proved that if $\Psi: C \times C \rightarrow C, \Psi \in\{+, \cdot, \min , \max \}$, then the set $\Psi^{-1}(E)$ is residual whenever $E$ is residual in $C$. In this paper we show that the operation of superposition $\Phi: C \times C^{*} \rightarrow C, \Phi(f, g)=f \circ g$, does not have an analogous property. For $D \subset C, E \subset C^{*}$, we abbreviate $\Phi(D \times E)=D \circ E$.

We start with an example illustrating a more general theorem. Fix two disjoint closed nondegenerate intervals $I, J \subset[0,1]$ and put

$$
A_{I J}=\left\{f \in C: \sup _{x \in I} f(x) \neq \sup _{x \in J} f(x)\right\} .
$$

It is known (see [3, Ch. 13, §3]) that $A_{I J}$ is an open dense subset of $C$. Indeed, $A_{I J}$ is open since if $g \in A_{I J}$ then $B(g, \varepsilon / 2) \subset A_{I J}$ when $\varepsilon=\mid \sup _{x \in I} g(x)-$ $\sup _{x \in J} g(x) \mid$. It is also dense since we can find a polygonal function $l$ in $A_{I J}$ (we use the fact that the set of all polygonals is dense in $C$ ).

Example 1. Let us define continuous functions $g:[0,1] \rightarrow[0,1], f:[0,1] \rightarrow$ $\mathbb{R}$ as follows

$$
g(x)=\left\{\begin{array}{lll}
1 / 4 & \text { for } & x \in[0,1 / 4) \cup(3 / 4,1] \\
x & \text { for } & x \in[1 / 4,3 / 8] \\
1-x & \text { for } & x \in[5 / 8,3 / 4] \\
3 / 8 & \text { for } & x \in(3 / 8,5 / 8)
\end{array}\right.
$$




$$
f(x)=\left\{\begin{array}{lll}
0 & \text { for } & x \in[0,9 / 32] \cup[11 / 32,1], \\
32 x-9 & \text { for } \quad x \in(9 / 32,10 / 32), \\
-32 x+11 & \text { for } \quad x \in[10 / 32,11 / 32) .
\end{array}\right.
$$

Define also $\varepsilon=1 / 32$ and $I=[1 / 4,3 / 8], J=[5 / 8,3 / 4]$. Let $h \in B(f, \varepsilon) \circ$ $B_{*}(g, \varepsilon)$. Then $h=f_{1} \circ g_{1}$ for some $f_{1} \in B(f, \varepsilon)$ and $g_{1} \in B_{*}(g, \varepsilon)$. By the definition of $g$ and $\varepsilon$ it is easy to see that

$$
[9 / 32,11 / 32] \subset g_{1}(I), \quad[9 / 32,11 / 32] \subset g_{1}(J) .
$$

By the definition of $f$ and $\varepsilon$, any function from $B(f, \varepsilon)$ achieves its supremum on $[0,1]$ at some point of the interval $[9 / 32,11 / 32]$. Hence by $(1)$ we infer that

$$
\sup _{x \in I} h(x)=\sup _{y \in[9 / 32,11 / 32]} f_{1}(y)=\sup _{x \in J} h(x),
$$

which means that $h \in C \backslash A_{I J}$. We have just proved the inclusion

$$
B(f, \varepsilon) \circ B_{*}(g, \varepsilon) \subset C \backslash A_{I J},
$$

which gives us

$$
B(f, \varepsilon) \times B_{*}(g, \varepsilon) \subset\left(C \times C^{*}\right) \backslash \Phi^{-1}\left(A_{I J}\right) .
$$

Therefore the preimage $\Phi^{-1}\left(A_{I J}\right)$ is not residual in $C \times C^{*}$.

Theorem 1. Any set of the form

$$
A_{I J}=\left\{f \in C: \sup _{x \in I} f(x) \neq \sup _{x \in J} f(x)\right\},
$$

where $I, J \subset[0,1]$ are nondegenerate disjoint closed intervals, is dense open in $C$, and its preimage $\Phi^{-1}\left(A_{I J}\right)$ under the operation of superposition $\Phi: C \times$ $C^{*} \rightarrow C$ is not residual in $C \times C^{*}$.

Proof. Choose a function $g \in C^{*}$ so that the graphs of restrictions $g_{I}, g_{J}$ of $g$ to intervals $I, J$ (respectively) are straight lines with non-zero slope coefficients, and such that $g(I)=g(J)$. Then by the continuity of $g$ one can find $\sigma>0$ such that, for some nondegenerate interval $[a, b]$, the following condition holds

$$
\left(\underset{g_{1} \in B_{*}(g, \sigma)}{\forall}\right)\left([a, b] \subset g_{1}(I) \wedge[a, b] \subset g_{1}(J)\right) .
$$

Next, choose $f \in C$ and $\varepsilon>0$ so that the condition

$$
\left(\underset{f_{1} \in B(f, \varepsilon)}{\forall}\right) \sup _{y \in[0,1]} f_{1}(y)=\sup _{y \in[a, b]} f_{1}(y)
$$

is fulfilled. (See Example 1, where $f$ is constructed for $[a, b]=[9 / 32,11 / 32]$.) Let $h \in B(f, \varepsilon) \circ B_{*}(g, \sigma)$. Then $h=f_{1} \circ g_{1}$ for some $f_{1} \in B(f, \varepsilon)$ and $g_{1} \in$ $B_{*}(g, \sigma)$. Hence by $(2)$ and (3) we have

$$
\sup _{x \in I} h(x)=\sup _{x \in I} f_{1}\left(g_{1}(x)\right)=\sup _{y \in[a, b]} f_{1}(y)
$$

and, analogously,

$$
\sup _{x \in J} h(x)=\sup _{x \in J} f_{1}\left(g_{1}(x)\right)=\sup _{y \in[a, b]} f_{1}(y) .
$$


That is why $\sup _{x \in I} h(x)=\sup _{x \in J} h(x)$, and $h \in C \backslash A_{I J}$. So we have proved the inclusion

$$
B(f, \varepsilon) \circ B_{*}(g, \sigma) \subset C \backslash A_{I J},
$$

which implies that

$$
B(f, \varepsilon) \times B_{*}(g, \sigma) \subset \Phi^{-1}\left(C \backslash A_{I J}\right)=\left(C \times C^{*}\right) \backslash \Phi^{-1}\left(A_{I J}\right) .
$$

Therefore $\Phi^{-1}\left(A_{I J}\right)$ is not residual in $C \times C^{*}$.

Remark 1. The preimage of $A_{I J}$ considered in Theorem 1 is of the second category, because it is open (as the preimage of an open set under a continuous mapping) and nonempty since $(i d, i d) \in \Phi^{-1}\left(A_{I J}\right)$, where $i d$ denotes the identity function on $[0,1]$.

In connection with Remark 1 a natural question arises whether the preimage of any residual set in $C$ under the operation of superposition is of the second category in $C \times C^{*}$. As we shall show in the next theorem, the answer is "no".

Theorem 2. Let $L$ denote the family of all pairs of intervals $(I, J)$ such that $I, J \subset[0,1]$ are nondegenerate, closed and disjoint, with rational endpoints. Then the preimage of a residual set $\bigcap_{(I, J) \in L} A_{I J}$ under the operation of superposition $\Phi: C \times C^{*} \rightarrow C$ is nowhere dense in $C \times C^{*}$.

Proof. Let $B(f, \varepsilon) \subset C$ and $B_{*}(g, \varepsilon) \subset C^{*}$. We shall show that there exist open balls $B\left(f_{1}, \sigma\right) \subset B(f, \varepsilon)$ and $B_{*}\left(g_{1}, \sigma\right) \subset B_{*}(g, \varepsilon)$ such that

$$
\left(B\left(f_{1}, \sigma\right) \times B_{*}\left(g_{1}, \sigma\right)\right) \cap \Phi^{-1}\left(\bigcap_{(I, J) \in L} A_{I J}\right)=\varnothing .
$$

Firstly, we choose in $B_{*}(g, \varepsilon)$ a function $g_{1}$ constant on some nondegenerate interval $[a, b] \subset[0,1]$; let for instance $g_{1}(x)=c$ for all $x \in[a, b]$. Without loss of generality we may assume that $c \in(0,1)$. Then there exists $\sigma_{1}>0$ such that $B_{*}\left(g_{1}, \sigma_{1}\right) \subset B_{*}(g, \varepsilon)$. Secondly, pick a function $f_{1}$ in $B(f, \varepsilon)$ such that for some number $\delta>0$ it is affine (i.e., of the form $x \mapsto a x+b$ ) increasing on $[c-\delta, c]$, and simultaneously affine decreasing on $[c, c+\delta]$. If necessary, we decrease the number $\sigma_{1}$ so that $\sigma_{1} \in(0, \delta]$. Then there exists $\varepsilon_{1}>0$ such that $B\left(f_{1}, \varepsilon_{1}\right) \subset B(f, \varepsilon)$ and

$$
\left\{\begin{array}{l}
f_{1}\left(c-\sigma_{1}\right)+\varepsilon_{1}<f_{1}(c)-\varepsilon_{1}, \\
f_{1}\left(c+\sigma_{1}\right)+\varepsilon_{1}<f_{1}(c)-\varepsilon_{1} .
\end{array}\right.
$$

By (4) and by the continuity of $f_{1}$ there exists $\sigma_{2} \in\left(0, \sigma_{1}\right)$ such that

$$
\left(\underset{\tilde{f} \in B\left(f_{1}, \varepsilon_{1}\right)}{\forall}\right) \sup _{y \in\left[c-\sigma_{1}, c+\sigma_{1}\right]} \tilde{f}(y)=\sup _{y \in\left[c-\sigma_{2}, c+\sigma_{2}\right]} \tilde{f}(y) .
$$

Let $I, J \subset[a, b]$ be such that $(I, J) \in L$. Choose a function $g_{2} \in B_{*}\left(g_{1}, \sigma_{1}\right)$ affine on intervals $I, J$, with non-zero slopes, such that for some number $\kappa>0$ the condition holds

$$
\left[c-\sigma_{2}-\kappa, c+\sigma_{2}+\kappa\right] \subset g_{2}(I)=g_{2}(J)
$$


for some number $\kappa>0$. Such a choice is possible since $\sigma_{2} \in\left(0, \sigma_{1}\right)$. If necessary, we decrease the number $\kappa$ so that $B_{*}\left(g_{2}, \kappa\right) \subset B_{*}\left(g_{1}, \sigma_{1}\right)$. Hence by (6) we have

$$
\left\{\begin{array}{l}
\left(\underset{\widetilde{g} \in B_{*}\left(g_{2}, \kappa\right)}{\forall}\right)\left[c-\sigma_{2}, c+\sigma_{2}\right] \subset \widetilde{g}(I) \subset\left[c-\sigma_{1}, c+\sigma_{1}\right], \\
\left(\underset{\widetilde{g} \in B_{*}\left(g_{2}, \kappa\right)}{\forall}\right)\left[c-\sigma_{2}, c+\sigma_{2}\right] \subset \widetilde{g}(J) \subset\left[c-\sigma_{1}, c+\sigma_{1}\right] .
\end{array}\right.
$$

Let $\sigma=\min \left\{\varepsilon_{1}, \kappa\right\}$. By (5) and (7) we see that if $(\tilde{f}, \widetilde{g}) \in B\left(f_{1}, \sigma\right) \times B_{*}\left(g_{2}, \sigma\right)$ then $\sup _{x \in I}(\widetilde{f} \circ \widetilde{g})(x)=\sup _{x \in J}(\widetilde{f} \circ \widetilde{g})(x)$. That is why $(\widetilde{f} \circ \widetilde{g}) \notin A_{I J}$. Consequently we have $(\widetilde{f}, \widetilde{g}) \notin \Phi^{-1}\left(A_{I J}\right)$.

Theorems 1 and 2 witness that the preimages of residual sets in $C$ under $\Phi$ can have a small Baire category size in $C \times C^{*}$. Of course the preimage of the whole $C$ is $C \times C^{*}$ - this is a trivial example where the preimage of a residual set is still residual. But, what is more surprising, there are nontrivial classical examples of that type. In [7, Proposition 3] we have proved that the set of all pairs $(f, g) \in C \times C^{*}$ with $f \circ g$ nowhere differentiable, is residual. This result can be slightly strengthened by the use of similar methods and the following simple criterion for preservation of the residuality by the preimages of continuous mappings.

Proposition 1. Let $X, Z$ be topological spaces and let $E \subset X$ be a residual set. If $\Phi: Z \rightarrow X$ is a continuous mapping such that the image $\Phi(U)$ is not nowhere dense for any nonempty open set $U \subset Z$, then $\Phi^{-1}(E)$ is a residual set.

Proof. By assumption, $X \backslash E=\bigcup_{i=1}^{\infty} A_{i}$, where all $A_{i}$ are all nowhere dense. Hence we have $\Phi^{-1}(X \backslash E) \subset \bigcup_{i=1}^{\infty} \Phi^{-1}\left(\operatorname{cl}\left(A_{i}\right)\right)$ and the last union is of the type $F_{\sigma}$, by the continuity of $\Phi$. Let $U_{i}=\operatorname{int}\left(\Phi^{-1}\left(\operatorname{cl}\left(A_{i}\right)\right)\right)$. Supposing that $U_{i} \neq \varnothing$, we have that $\Phi\left(U_{i}\right)$ is not nowhere dense, which contradicts $\Phi\left(U_{i}\right) \subset \operatorname{cl}\left(A_{i}\right)$. That is why $\Phi^{-1}(E)$ is the complement of the first category set.

Remark 2. If in Proposition 1 we additionally assume that $E$ is of type $G_{\delta}$, then the condition " $\Phi(U)$ is not nowhere dense for any nonempty open set $U \subset Z$ " can be replaced by " $\Phi(U) \cap E \neq \varnothing$ for any nonempty open set $U \subset Z$ ".

Proposition 2. The preimage of the residual set

$$
B^{+}=\left\{f \in C:(\underset{n \in \mathbb{N}}{\forall})(\underset{x \in[0,1-1 / n]}{\forall})(\underset{h \in(0,1-x)}{\exists})\left|\frac{f(x+h)-f(x)}{h}\right|>n\right\}
$$

(see [5, Ch. 11]) under the operation of superposition is residual in $C \times C^{*}$.

Proof. Since $B^{+}$is of type $G_{\delta}$, by Remark 2, in any open balls $B(f, \varepsilon) \subset$ $C, B_{*}(g, \varepsilon) \subset C^{*}$ it suffices to find functions $p, l$ (respectively) such that $p \circ l \in B^{+}$. One can do this by taking as $p$ any function from $B^{+} \cap B^{-} \cap B(f, \varepsilon)$ (where $B^{-}$is defined as below) and as $l$ any polygonal in $B_{*}(g, \varepsilon)$ with non-zero slopes. The details are similar to those in [7, Proposition 3]. 
Analogously, one can show that, by the operation of superposition, the preimage of the residual set

$$
B^{-}=\left\{f \in C:(\underset{n \in \mathbb{N}}{\forall})(\underset{x \in[1 / n, 1]}{\forall})(\underset{h \in(0, x)}{\exists})\left|\frac{f(x-h)-f(x)}{h}\right|>n\right\}
$$

is also residual in $C \times C^{*}$.

As a corollary we prove a result related to the well-known Banach-Mazurkiewicz theorem (see [2], [4], [5]).

Theorem 3. The set of all pairs $(f, g) \in C \times C^{*}$ such that at any point $x \in[0,1]$ there exist neither finite right-hand derivative $(f \circ g)_{+}^{\prime}(x)$ nor finite left-hand derivative $(f \circ g)_{-}^{\prime}(x)$, is residual in $C \times C^{*}$.

Proof. Consider the set $E^{+}$(respectively $\left.E^{-}\right)$of all pairs $(f, g) \in C \times C^{*}$ such that at any point $x \in[0,1)$ (respectively $x \in(0,1]$ ) there does not exist a finite right-hand derivative $(f \circ g)_{+}^{\prime}(x)$ (respectively, a finite left-hand derivative $\left.(f \circ g)_{-}^{\prime}(x)\right)$. Then $E^{+}$(respectively $E^{-}$) is the preimage by $\Phi$ of a set containing $B^{+}$(respectively $B^{-}$). Taking into account Proposition 2 and the comment preceding Theorem 3, we infer that both sets $E^{+}$and $E^{-}$are residual in $C \times C^{*}$. Their intersection is also residual.

Let us finish with a similar consideration when $C$ is replaced by the space of homeomorphisms from $[0,1]$ onto $[0,1]$. Then the behaviour of preimages under $\Phi$ is more regular from the Baire category standpoint.

Denote by $H$ the space of all homeomorphisms from $[0,1]$ onto $[0,1]$, endowed with the sup norm (thus $H$ is topologically complete) and let $\Phi: H \times H \rightarrow H$ be the operation of superposition. Fix a function $f \in H$ and define $\Phi_{f}: H \rightarrow H$ by $\Phi_{f}(g)=\Phi(f, g), g \in H$. Since $(H, \Phi)$ is a topological group, $\Phi_{f}$ is a homeomorphism. Hence we infer that for any open balls $B_{1}, B_{2} \subset H$ the image $\Phi\left(B_{1} \times B_{2}\right)$ is nonempty and open in $H$. Proposition 1 implies that $\Phi^{-1}(E)$ is residual in $H \times H$, whenever $E$ is residual in $H$. We obtain similar results for the operations of superpositions $\Phi: C \times H \rightarrow C$ and $\Phi: H \times C^{*} \rightarrow C^{*}$. This contrasts with our Theorems 1 and 2 .

\section{ACKNOWLEDGEMENTS}

I would like to thank Marek Balcerzak for his valuable comments. I am grateful to the Referee who suggested some improvements.

\section{REFERENCES}

1. M. Balcerzak, A. Wachowicz, and W. Wilczyński, Multiplying balls in the space of continuous functions on [0,1]. Preprint 2004/10, Faculty of Mathematics, University of $€ o ́ d z ́$ (available also on the World Wide Web at the URL: http://www.math.uni.lodz.pl/preprints).

2. S. BANACH, Über die Baire'sche Kategorie gewisser Funktionenmengen. Studia Math. 3(1931), 174-179. 
3. A. Bruckner, Differentiation of real functions. Second edition. CRM Monograph Series, 5. American Mathematical Society, Providence, RI, 1994.

4. S. Mazurkiewicz, Sur les fonctions non derivables. Studia Math. 3(1931), 92-94.

5. J. C. Охтову, Measure and category. Graduate Texts in Mathematics, Vol. 2. SpringerVerlag, New York-Berlin, 1971.

6. A. Wachowicz, On some residual sets. (Polish) Ph.D. dissertation, Eódź Technical University, Łódź, 2004.

7. A. WACHOWICZ, Baire category and standard operations on pairs of continuous functions. Tatra Mt. Math. Publ. 24(2002), part II, 141-146.

(Received 1.03.2005; revised 27.04.2005)

Author's address:

Łódź Technical University, Institute of Mathematics

ul. Wólczańska 215, 93-005 Łódź

Poland

E-mail: aw314@poczta.fm 